\title{
Functional Anatomical Evidence for Respiratory Rhythmogenic Function of Endogenous Bursters in Rat Medulla
}

\author{
Nicholas M. Mellen and Deepak Mishra \\ Kosair Children's Hospital Research Institute, University of Louisville, Louisville, Kentucky 40202
}

\begin{abstract}
Endogenous burster neurons (EBs) have been found at the level of the facial nucleus (VIIn), and $500 \mu \mathrm{m}$ caudally, within the preBötzinger complex (preBötC). They have been proposed as either causal to or playing no role in respiratory rhythmogenesis. Little is known about their broader distribution in ventrolateral medulla. Here, $\mathrm{a} \mathrm{Ca}^{2+}$ indicator was used to record respiratory network activity in ventrolateral medulla, and, following synaptic blockade, to identify EBs active at perfusate $\mathrm{K}^{+}$concentrations $\left(\left[\mathrm{K}^{+}\right]_{\mathrm{o}}\right)$ of 3,6 , and 9 mм. Recordings were made along the respiratory column, extending $300 \mu \mathrm{m}$ rostrally, and $1100 \mu \mathrm{m}$ caudally from the caudal pole of VIIn (VIIc), in the in vitro tilted sagittal slab preparation, isolated from neonate male and female Sprague Dawley rats. Activity under matching $\left[\mathrm{K}^{+}\right]_{\mathrm{o}}$ in the intact respiratory network was subsequently investigated. Respiratory neurons $(n=401)$ formed statistically significant clusters at the VIIc, within the preBötC, and $100 \mu \mathrm{m}$ caudal to the preBötC. EBs $(n=693)$ formed statistically significant clusters that overlapped with respiratory clusters at the VIIc and preBötC. EB activity increased significantly as $\left[\mathrm{K}^{+}\right]_{0}$ was increased, as did neurons that remained coupled following synaptic blockade. The overlap between respiratory and EB clusters in regions of ventrolateral medulla identified as rhythmogenic supports the hypothesis that EBs are constituents of rhythmogenic networks. In addition, the observation of truncated inspiratory bursts and ectopic bursting in respiratory neurons when $\left[\mathrm{K}^{+}\right]_{0}$ was elevated in the intact network is consistent with a causal role for EBs in respiratory rhythmogenesis.
\end{abstract}

\section{Introduction}

In mammals, circuits essential for respiratory rhythmogenesis are distributed bilaterally as columns in ventrolateral medulla. These networks remain spontaneously active in a variety of neonate rodent in vitro medullary preparations (Suzue, 1984; Smith and Feldman, 1987; Smith et al., 1991). Two anatomical regions have been shown to have rhythmogenic function in vitro: the retrotrapezoid nucleus/parafacial respiratory group (RTN/pFRG), ventral and caudal to the facial nucleus (VIIn) (Onimaru et al., 2009), and the pre-Bötzinger complex (preBötC), $500 \mu \mathrm{m}$ caudal to the caudal pole of the VIIn (VIIc) (Ruangkittisakul et al., 2006, 2008). Both these regions are rich in endogenous bursters (EBs), which display periodic bursting in the absence of synaptic input (Johnson et al., 1994; Onimaru et al., 1995). EBs have been proposed to play a critical role in respiratory rhythm generation, elaborated using cellular (Butera et al., 1999a) and network (Butera et al., 1999b) models, which have obtained experimental support (Del Negro et al., 2001). More recently, it has been shown that EBs are not necessary for respiratory rhythmogenesis (Del Negro et al., 2002a), consistent with the conjecture that respiratory rhythm is generated by excitatory networks of neurons whose bursting properties are irrelevant to their rhythmogenic

\footnotetext{
Received Nov. 6, 2009; revised April 21, 2010; accepted May 10, 2010.

This work was supported by National Institutes of Health Grant HL06800. We thank Dr. Consuelo Morgado-Valle for comments on this manuscript.

Correspondence should be addressed to Nicholas Mellen, Kosair Children's Hospital Research Institute, University of Louisville, 570 South Preston Street, Baxter Building 1, Suite 304, Louisville, KY 40202. E-mail: nicholas. mellen@louisville.edu.

DOI:10.1523/JNEUROSC1.5510-09.2010

Copyright $\odot 2010$ the authors $\quad 0270-6474 / 10 / 308383-10 \$ 15.00 / 0$
}

function (Rekling and Feldman, 1998). This hypothesis has gained empirical support (Del Negro et al., 2002a; Pace et al., 2007; Pace and Del Negro, 2008), and has been elaborated in models (Kosmidis et al., 2004; Rubin et al., 2009). Thus, the role of EBs in respiratory rhythmogenesis continues to be debated.

Although others have characterized EBs within identified respiratory rhythmogenic networks using optical methods (Koshiya and Smith, 1999; Thoby-Brisson et al., 2009), typically, EBs have been studied using single-unit recordings. Because definitive EB identification is obtained following blockade of synaptic transmission, only one burster can typically be identified per experiment. Thus, estimates of the number and distribution of EBs along the respiratory column are lacking. Based on these necessarily sparse data, the functional role of EBs has been difficult to assess.

Here, the overlap between respiratory networks and EBs was characterized. Optical recordings were made from the surface of the sagittal slab preparation (Barnes et al., 2007), along the respiratory column under control conditions, and following synaptic blockade at physiological ( $3 \mathrm{~mm})$ and elevated (6 mM, $9 \mathrm{~mm})$ $\left[\mathrm{K}^{+}\right]_{\mathrm{o}}$. Because this approach allowed us to sample activity from the network at the exposed surface of the slice, the incidence, distribution, and extent of overlap between respiratory networks and EBs could be assessed directly. We report three main findings: (1) EBs form clusters along ventrolateral medulla; (2) clusters of respiratory neurons and clusters of EBs only overlap at the VIIc and in the rostral preBötC, suggesting that $\mathrm{EB}$ properties reflect a functional specialization that contributes to the generation and regulation of respiratory rhythm; and (3) there are relatively low EB numbers at physiological $\left[\mathrm{K}^{+}\right]_{\mathrm{o}}$, suggesting that under conditions of low respiratory drive, EBs that are subthreshold to bursting may 
amplify synaptic inputs via the same nonlinear membrane properties that generate bursting at more depolarized levels (Ramirez et al., 2004; Feldman and Del Negro, 2006), while active EBs may directly contribute to rhythmogenesis.

\section{Materials and Methods}

Dissection. In accordance with methods approved by the Institutional Animal Care and Use Committee, neonate male and female rat pups (postnatal day $0-3$ ) were anesthetized with isoflurane. The brainstem (transected just rostral to VIIn) and spinal cord were isolated using standard methods (Smith and Feldman, 1987) in a dissection chamber perfused with chilled $\left(5^{\circ} \mathrm{C}\right)$ artificial CSF (aCSF) containing the following (in mM): $128.0 \mathrm{NaCl}, 3.0 \mathrm{KCl}$, $1.5 \mathrm{CaCl}_{2}, 1.0 \mathrm{MgSO}_{4}, 21.0 \mathrm{NaHCO}_{3}, 0.5 \mathrm{NaH}_{2} \mathrm{PO}_{4}$, and 30.0 glucose, equilibrated with $95 \% \mathrm{O}_{2}-5 \% \mathrm{CO}_{2}$ (carbogen).

A tilted sagittal slab preparation was cut at a compound angle of $2.2^{\circ}$ rostrocaudal, and $12^{\circ}$ ventrodorsal tilt, using a device developed for this purpose (Mellen, 2008). Level of section was determined as a ratio of brainstem width: respiratory networks were exposed by cutting at 0.34 of the distance from the midline to the lateral edge of the preparation; the blind side was cut at $0.7-0.8$ of the full width of the brainstem. Although small variations between preparations are unavoidable, because of both experimenter error and biological variability, this variation must be small for the preparation to remain viable: preparations cut too medially fail to generate a stable rhythm, while preparations cut too laterally fail to generate optical signals.

The preparation was then mounted on the narrow edge of a 2-mmthick semicircular inert elastomer slab (Sylgard, Dow-Corning) and incubated for $1 \mathrm{~h}$ in a carbogenated solution containing the low-affinity $\left(K_{\mathrm{D}}=1.86 \mu \mathrm{M}\right)$ cell-permeant $\mathrm{Ca}^{2+}$ indicator fluo-8L AM (ABDBioquest; $50 \mu \mathrm{g}$ ), $10 \mu \mathrm{l}$ of a $20 \%$ solution of the surfactant pluronic F-127 (Invitrogen), dissolved in DMSO, to which $750 \mu \mathrm{l}$ of aCSF was added for a final concentration of $60 \mu \mathrm{M}$. After dye loading, the dye-loaded preparation was transferred to a recording chamber $(\sim 2$ ml volume; JG 23 W/HP, Warner Instruments), mounted on an upright microscope (Axioskop 2, Zeiss Instruments), and perfused (4 $\mathrm{ml} / \mathrm{min}$ ) with carbogenated aCSF maintained at $27^{\circ} \mathrm{C}$.

Data acquisition. A suction electrode connected to a differential amplifier (EXT-01C/DPA 2F; NPI Electronics) was placed on ventral root $\mathrm{C} 1$, and the population recording was digitized at $1 \mathrm{kHz}$ (PCI-MIO16XE-10, National Instruments). Fluorescence images were obtained using a cooled $\left(-80^{\circ} \mathrm{C}\right)$ CCD camera (EM-CCD 9100-13, Hamamatsu), connected to a frame grabber (IMAQ PCI-1422, National Instruments), and illuminated using a shuttered xenon arc lamp (Lambda DG-4, Sutter Instruments). Low-magnification $(10 \times)$ images of the preparation were obtained to detect regions of optical activity, and to obtain reference images for the alignment of high-magnification $(20 \times)$ image series used during experimental protocols. In experiments in which respiratory networks were recorded before and during synaptic blockade, exposure times were minimized by recording at $20 \times$ in $2 \times 2$ binning mode (pixels $=1.6 \times 1.6 \mu \mathrm{m})$, which permitted brief exposures $(\leq 20 \mathrm{~ms})$, sampled at $3 \mathrm{~Hz}$. Thus, over the course of an experiment, total light exposure did not exceed $12 \mathrm{~s}$, thereby minimizing photobleaching and phototoxicity. In network-intact $\left[\mathrm{K}^{+}\right]_{\mathrm{o}}$ manipulation experiments, the sampling rate was increased to $10 \mathrm{~Hz}$ so as to be able to resolve ectopic bursting; because these experiments involved a total of $300 \mathrm{~s}$ of recordings, total exposure time was $20 \mathrm{~s}$. Because lipophilic indicators only label cells within $40 \mu \mathrm{m}$ of the slice surface (Funke et al., 2007), only superficial cells were recorded from.

Experimental protocol. Overlapping low-magnification $(10 \times)$ image series were obtained at $3 \mathrm{~Hz}$ for $40-60 \mathrm{~s}$ to detect regions of respirationmodulated neuronal activity. Identified respiratory networks were then recorded at high magnification $(20 \times)$ for $40-60 \mathrm{~s}$ at $3 \mathrm{~Hz}$ in $2 \times 2$ binning mode. EB activity was detected by recording from the same regions from which respiratory network activity had been sampled, following blockade of fast synaptic transmission using a cocktail consisting of the AMPA receptor blocker CNQX $(20 \mu \mathrm{M})$, the NMDA receptor blocker APV $(20 \mu \mathrm{M})$, the $\mathrm{GABA}_{\mathrm{A}}$ receptor blocker bicuculline $(10 \mu \mathrm{M})$, and the glycine receptor blocker strychnine ( $1 \mu \mathrm{M}$; all drugs from Sigma-
Aldrich). This cocktail was dissolved in aCSF containing 3, 6, and $9 \mathrm{~mm}$ $\left[\mathrm{K}^{+}\right]_{\mathrm{o}}$. The presentation order of each $\left[\mathrm{K}^{+}\right]_{\mathrm{o}}$ solution was randomized to minimize the bias associated with slow-time course changes in recording conditions, such as preparation run-down, photobleaching, and dye sequestration. In each experiment, optical recordings were performed from up to two locations with little or no overlap. A digital translation stage (MT-2000, Sutter Instruments) that provided recording location coordinates permitted reproducible repositioning from one location to the other. Optical recordings were initiated $5 \mathrm{~min}$ after cessation of motor output, and $3 \mathrm{~min}$ following changes in $\left[\mathrm{K}^{+}\right]_{\mathrm{o}}$ under continued synaptic blockade; in subsequent experiments in which synaptically coupled networks were recorded under 3,6 , and $9 \mathrm{~mm}\left[\mathrm{~K}^{+}\right]_{0}$, equilibration time was extended to $10 \mathrm{~min}$. Because recordings were made from up to two locations, under four conditions, with delays associated with wash-in and repositioning, a balance had to be struck between recording duration, and slow time course changes associated with and dye sequestration (Thomas et al., 2000). In light of these constraints, recording epochs under synaptic blockade conditions lasted no less than $133 \mathrm{~s}$ and no more than $167 \mathrm{~s}$ (400-500 frames).

Signal processing. $\mathrm{Ca}^{2+}$ transients associated with neuronal activity were extracted from each image series using semiautomated methods implemented in LabView (National Instruments) performed in three stages: region of interest (ROI) generation, signal extraction, and manual screening (Mellen and Tuong, 2009). Single-wavelength $\mathrm{Ca}^{2+}$ indicators such as fluo-8L used here provide information about relative rather than absolute changes in $\left[\mathrm{Ca}^{2+}\right]_{\mathrm{i}}$; in addition, although fluo-8L has relatively low $\mathrm{Ca}^{2+}$ affinity $\left(K_{\mathrm{D}}=1.86 \mu \mathrm{M}\right)$, signal decay reflects not only neuronal activity, but also dye- $\mathrm{Ca}^{2+}$ binding kinetics. As a consequence, although qualitative changes in $\mathrm{Ca}^{2+}$ signals may be interpretable, generally, only event times associated with optical trace peaks are extracted for subsequent analysis.

Traces were subjected to high-pass filtering: each trace was subjected to a $14 \mathrm{~s}$ moving average low-pass filtering to eliminate physiologically interesting transients from the signal. High-pass filtering was then obtained by subtracting the low-pass filtered signal from the raw signal, and the average of this low-pass filtered signal was used as our estimator of static fluorescence $F$, which was used to scale changes in relative intensity $\Delta F$. Finally, to facilitate accurate peak detection, jitter associated with fluctuations in arc-lamp intensity was reduced by applying a two-frame moving average to the high-pass filtered trace. Peaks were then extracted using trough-to-peak amplitude. A quadratic fit algorithm was then applied to extract peak times, and dots corresponding to peak locations were superimposed on optical traces to permit validation of peak selection parameters. Because the moving average did not uniformly filter data at the beginning and end of datasets, spurious peaks associated with unfiltered data were eliminated from the beginning and end of each trace.

Trace selection. Identification of respiration-modulated neurons was achieved by inspecting traces superimposed on root activity, and based on the burst-triggered average of the optical trace, which in the case of respiration-modulated neurons showed a well defined peak or trough in relation to the averaged inspiratory burst. EBs were selected in two stages: first all traces obtained under conditions of synaptic blockade showing time-varying fluctuations in brightness were included. Thereafter, only traces whose peak-to-peak periods had a coefficient of variation $(\mathrm{CV}) \leq$ 0.50 were retained.

Image alignment and collation. For each experiment, high-magnification images and their associated ROIs were aligned to the low-magnification images obtained at the experiment's outset (Photoshop; Adobe Systems) based on anatomical and morphological matches. From each aligned set of images, line drawings were extracted consisting of the following: (1) outlines (drawn by hand) of the brainstem's ventral surface and the VIIn, which could be identified by banding and larger somata within the VIIn, and by densely packed, smaller cells along its border; and (2) ROIs generated under control conditions, and following synaptic blockade, under each $\left[\mathrm{K}^{+}\right]_{\mathrm{o}}$ condition. These line drawings were exported to Illustrator (Adobe Systems). All sequentially recorded high-magnification recordings were brought into register with each other based on anatomical and morphological matches, so that neurons active under more than one condition could be identified based on the overlap of their ROIs, and their associated traces could be indexed as 


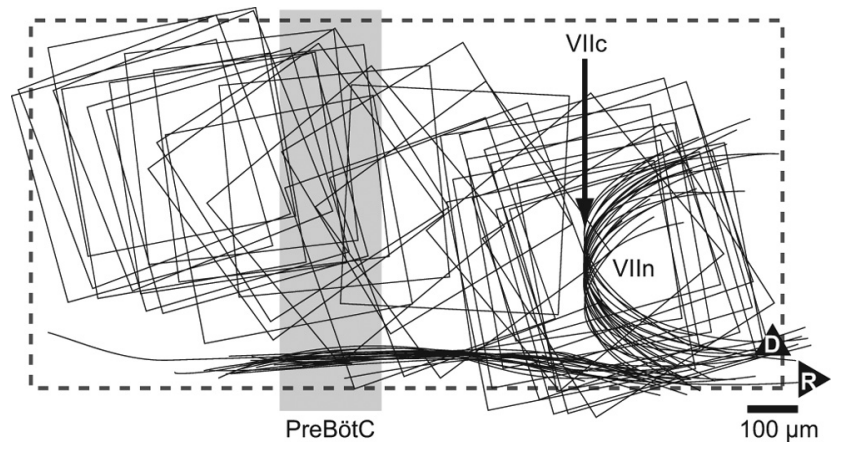

Figure 1. Optical recording locations extend $1100 \mu \mathrm{m}$ caudal from the VIIC (indicated by arrow). Each box represents the location of sequential optical recording epochs. The bounding rectangle (dashed line) represents the area over which spatial statistics were carried out. D, Dorsal; $R$, rostral.

sharing a common source. To collate data across experiments, an Illustrator file consisting of five layers was generated: the first contained the anatomical outlines of the ventral surface and the VIIn, and subsequent layers contained dots aligned to ROIs color coded according to recording condition (control, 3,6 , and $9 \mathrm{~mm}\left[\mathrm{~K}^{+}\right]_{\mathrm{o}}$ ). Data across experiments were then aligned using the VIIc and the ventral surface within a common coordinate frame that spanned all recording locations (Fig. 1, dashed box). In-house software was then used to extract dot coordinates from each dataset's aligned dot diagram, based on dot color, for analysis using spatial statistical methods. Because the VIIc shows little mediolateral taper, the rostrocaudal location of VIIc used here to align datasets is a reliable landmark.

Identification of coupled neurons following fast synaptic transmission blockade. Because of the low sampling rates used here, cross-correlation could not be used to characterize coupling between respiratory network constituents in the intact network. Under conditions of synaptic blockade, however, cross-correlation was used to identify neurons that remained coupled despite blockade of fast synaptic transmission. Coupling was treated as a binary variable, with the mean of cross-correlation coefficients obtained from all pairwise comparisons between respirationmodulated neurons $\left(R^{2}=0.67\right)$ as the threshold for classifying EBs as coupled. Coupling following fast synaptic transmission blockade was tested for between all EBs in every dataset.

Spatial statistics. To test whether EBs and respiratory neurons show complete spatial randomness (CSR) in their distribution, the $\chi^{2}$ test based on $100 \mu \mathrm{m} \times 100 \mu \mathrm{m}$ quadrat counts was used. In addition, we tested for the presence of statistically significant clusters by using the Dirichlet (or Voronoi) tessellation method, a measure to detect spatial clustering, in which for a given point $p$ in the pattern $X$, a tile is drawn that contains the region of space closer to $p$ than to any other point of $X$ (Okabe et al., 1992; Duyckaerts and Godefroy, 2000). Thus, for points in clusters, the associated tiles are small. Statistically significant clusters were identified by comparing Dirichlet tile sizes from the actual data to tile areas obtained from surrogate datasets in which point coordinates from the original dataset were randomized using the quadratureresample command, which creates a resampled point pattern by dividing the bounding rectangle enclosing all points in the dot diagram (Fig. 1, dotted box) into rectangular quadrats and randomly resampling the list of quadrats, followed by the rjitter command, which randomly displaced each point in the dataset. Because the logarithm of tile sizes from surrogate datasets approximated a normal distribution, estimates of the $95 \%$ confidence interval (CI) for log tile sizes from randomized distributions were obtained from 10 surrogate datasets, and points associated with contiguous Dirichlet tiles obtained from the actual data points whose logarithmically transformed tile size was smaller than the $95 \%$ CI of the surrogate dataset tile sizes were considered constituents of statistically significant clusters (supplemental Fig. 1, available at www.jneurosci.org as supplemental material). All analyses were performed using the spatstat module of the open-source statistics package R (Baddeley and Turner, 2005).

Descriptive statistics and tests for statistical significance of cell counts and respiratory periods were performed using R and Origin (OriginLab); data are reported as mean \pm SEM, except in cases when data are skewed; then the median is reported.

\section{Results}

In 23 experiments, a total of 35 locations were sampled over consecutive epochs, both under control conditions and following synaptic blockade, at 3, 6, and $9 \mathrm{~mm}\left[\mathrm{~K}^{+}\right]_{0}$, in randomized presentation order. In 12/23 experiments, recordings at two locations were carried out; in the remaining $11 / 23$ postnatal day 0 to postnatal day 3 preparations, sequential recordings were made from only one location. Recordings extended $300 \mu \mathrm{m}$ rostrally, and $1100 \mu \mathrm{m}$ caudally from the VIIc along ventrolateral medulla, covering a region including, but not limited to, the preBötC and the RTN/pFRG (Fig. 1). In total, 693 EBs were recorded from, as well as 401 respiratory neurons, of which $24 \%$ (97/401) were also EBs. Although the low sampling rates used here preclude detailed classification of respiratory neurons based on firing phase, tonically active expiratory neurons silent only during inspiration could unambiguously be identified, and constituted $16 \%$ of the respiratory neuron sample (64/401), double the frequency reported in the embryonic mouse en bloc preparation (Eugenin et al., 2006). Subsequently, another seven experiments from synaptically coupled networks at 3,6 , and $9 \mathrm{~mm}\left[\mathrm{~K}^{+}\right]_{\mathrm{o}}$ were performed from respiratory networks at VIIc $(n=3)$ and at the preBötC $(n=4)$; a total of 405 respiratory neurons were recorded, of which $37 \%(151 / 405)$ were active at more than one $\left[\mathrm{K}^{+}\right]_{\mathrm{o}}$.

\section{Respiration-modulated EBs and EB coupling detected following synaptic blockade}

In a representative experiment, respiratory networks (Fig. $2 A$, white ROIs) were recorded at the VIIc (4 cells), and $500 \mu \mathrm{m}$ caudally, at the level of the preBötC ( 7 cells). Because respiratory neurons could robustly be identified by comparison to motor output traces recorded from $\mathrm{C} 1$, control activity was only sampled for 40-60 s (Fig. 2B). Respiratory neurons had high CVs (Fig. $2 B$, to the left of traces), because of both skipped cycles (Fig. $2 B$, shaded box) and ectopic bursts (i.e., neuronal activity unaccompanied by inspiratory motor output) (Fig. $2 B$, vertical arrows). In this dataset, EBs were found at $3 \mathrm{~mm}$ only at the level of the preBötC (Fig. $2 \mathrm{~A}$, blue ROIs), but at $6 \mathrm{~mm}$ (Fig. $2 \mathrm{~A}$, green ROIs) and $9 \mathrm{~mm}$ (Fig. $2 A$, red ROIs), EBs were found in both recording locations. In this experiment, three of the respirationmodulated neurons at the VIIc were found to be EBs following synaptic blockade; of these, one was active at both 3 and $6 \mathrm{~mm}$ $\left[\mathrm{K}^{+}\right]_{\mathrm{o}}$. In addition, one nonrespiratory EB was active at both 6 and $9 \mathrm{~mm}\left[\mathrm{~K}^{+}\right]_{\mathrm{o}}$ (traces associated with a single unit's activity are linked by fine lines in Fig. $2 B$ ).

Because EBs were recorded optically, and thus sampled in parallel, coupling that persisted despite fast synaptic transmission blockade could be detected. Because many bursters had similar frequencies, even if uncoupled, they appear to align because of slowly drifting phase relations. Coupling could be identified by cross-correlation analysis. In this dataset, only two pairs of EBs met or exceeded the threshold obtained from cross-correlation analysis of respiration-modulated neurons in the coupled networks (Fig. $2 \mathrm{~B}$, heavier traces), one pair at the level of the preBötC under $9 \mathrm{~mm}\left[\mathrm{~K}^{+}\right]_{\mathrm{o}}\left(R^{2}=0.78\right)$, the other at VIIc under 6 $\mathrm{mm}\left[\mathrm{K}^{+}\right]_{\mathrm{o}}\left(R^{2}=0.86\right)$. In these pairs, the phase relationship between optical traces is constant, but in addition, aperiodic fluctuations in burst envelope and bursting frequency are reproduced in both traces of each pair, consistent with coupling. Because the ROIs from which these traces were obtained are at a distance from one another (linked by dotted lines with arrow- 
A

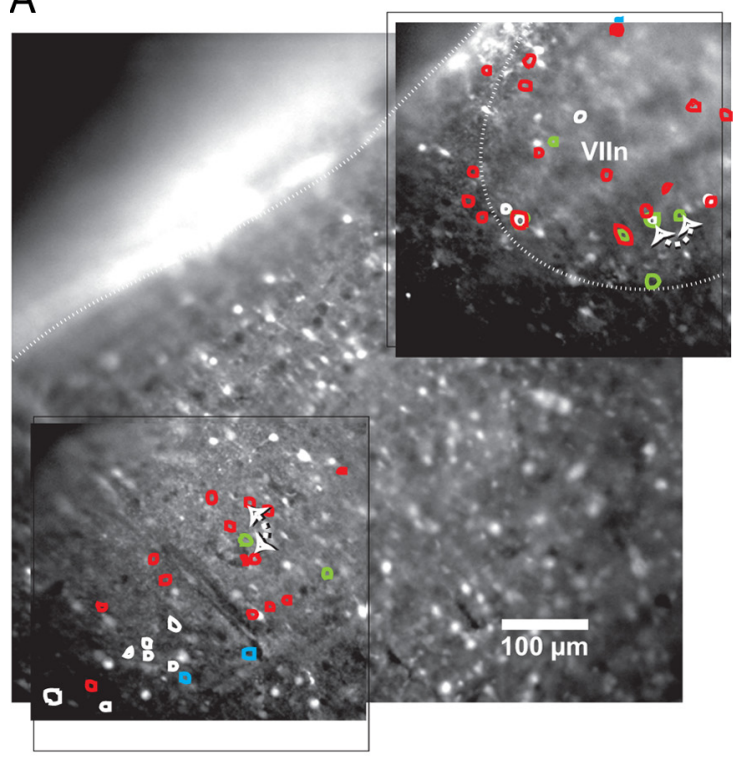

B

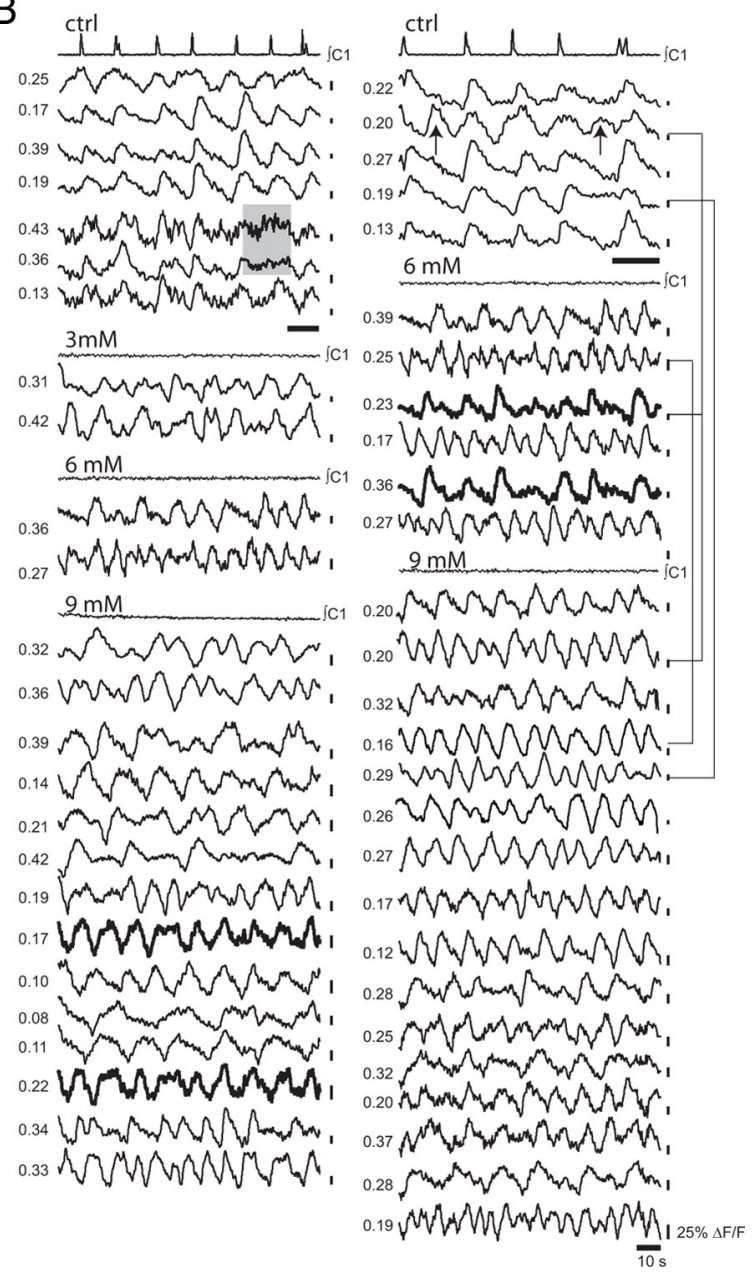

Figure 2. Optical recordings of respiratory neurons and EBs obtained at the VIIIn and $500 \mu \mathrm{m}$ caudally. $A$, View of the preparation, with respiratory neuron ROls in the intact network (white outlines) and EB ROls active at $\left[\mathrm{K}^{+}\right]_{0}$ of $3 \mathrm{~mm}$ (blue outlines), $6 \mathrm{~mm}$ (green outlines), and $9 \mathrm{~mm}$ (red outlines) following blockade of fast synaptic transmission. Black dotted lines with arrowheads at both ends link neurons that remained coupled following synaptic blockade. $\boldsymbol{B}$, Ventral root $\left(\int C 1\right)$ and optical recordings associated with successive recordings from the caudal (left column) and rostral (right column) recording locations, under control conditions, and following synaptic blockade, at successively higher $\left[\mathrm{K}^{+}\right]_{0}$ (indicated just above heads, Fig. $2 A$ ), it is unlikely that these closely matched traces were generated by a common neuron.

Despite the low sampling rates used here, optical recordings revealed considerable EB heterogeneity. In some cells, bursts shared the steep rise and exponential roll-off common to respiration-modulated neurons, while others had symmetric saw-tooth or rounded burst envelopes; further, some cells showed burst-to-burst variability in amplitude, whereas others had highly stereotypic burst amplitudes and envelopes.

\section{Pooled data reveal that EB numbers, but not EB bursting frequency, increase with $\left[\mathrm{K}^{+}\right]_{\mathrm{o}}$}

Respiratory neuron periods (median $=6.8 \mathrm{~s}$ ) measured before synaptic blockade (Fig. 3Ai) were significantly shorter $(p<0.01$, Wilcoxon ranked means test) than EB periods (median $=11.7 \mathrm{~s}$ ); this difference is apparent in the relatively small overlap between the histograms of respiratory periods (Fig. $3 B$, solid gray) and EB periods (Fig. $3 B$, black line). By inspection, respiratory neurons appear to have more variable periods than EBs. On one hand, respiratory neurons were identifiable by comparison with motor output; thus, cells that failed to fire in a given respiratory cycle, and/or that fired in the absence of motor output, could nonetheless be identified as respiratory neurons despite having variable periods. On the other hand, because all neurons with a $\mathrm{CV}>0.5$ were excluded from this study, the variability of the sample included in this study reflects the inclusion criteria used.

Mean respiratory and nonrespiratory EB periods at $3 \mathrm{~mm}$ $(13.0 \mathrm{~s} ; 13.3 \mathrm{~s})$ were not significantly slower than EB period at 6 $\mathrm{mm}(12.3 \mathrm{~s} ; 13.0 \mathrm{~s})$ or $9 \mathrm{~mm}(12.0 ; 12.6 \mathrm{~s} ; p>0.2)$ (Fig. $3 A i-A i v)$. At each $\left[\mathrm{K}^{+}\right]_{\mathrm{o}}$, mean period reflects the contribution of neurons active only in that recording epoch, as well as the contribution of neurons active in more than one recording epoch. Neurons active in multiple recording epochs were $26 \%(181 / 693)$ of EBs recorded from, but their contribution to mean EB period was greater than their numbers, since their activity at each $\left[\mathrm{K}^{+}\right]_{\mathrm{o}}$ generated a data point. Thus, for each $\left[\mathrm{K}^{+}\right]_{\mathrm{o}}$, approximately half of the EBs recorded from were active at more than one $\left[\mathrm{K}^{+}\right]_{\mathrm{o}}$ (Fig. 3C, gray bars). The lack of significant differences in period at different $\left[\mathrm{K}^{+}\right]_{\mathrm{o}}$ is consistent with the observation that slightly less than half of the neurons active at multiple $\left[\mathrm{K}^{+}\right]_{\mathrm{o}}$ showed a decrease in period at a more elevated $\left[\mathrm{K}^{+}\right]_{\mathrm{o}}$. Thus, the lack of significant differences between EB periods obtained at different $\left[\mathrm{K}^{+}\right]_{\mathrm{o}}$ reflects both the lack of consistent voltage dependence among EBs active at more than one $\left[\mathrm{K}^{+}\right]_{\mathrm{o}}$ and the homogeneity of bursting frequencies among EBs active at only one $\left[\mathrm{K}^{+}\right]_{\mathrm{o}}$ (Onimaru and Homma, 2008).

Of the 97 respiration-modulated EBs recorded from, 24 were active at $3 \mathrm{~mm}, 33$ at $6 \mathrm{~mm}$, and 40 at $9 \mathrm{~mm}\left[\mathrm{~K}^{+}\right]_{\mathrm{o}}$. The average number of respiration-modulated EBs per experiment was $2.8 \pm$ 2 (Fig. $3 C$, dark gray bar). In addition, $41 \%$ of these respirationmodulated EBs (40/97) were active at more than one $\left[\mathrm{K}^{+}\right]_{\mathrm{o}}$.

The average number of EBs per recording location increased with $\left[\mathrm{K}^{+}\right]_{\mathrm{o}}$, from $3 \mathrm{mM}(4.0 \pm 0.7$ cells; total $n=91)$, to $6 \mathrm{mM}$ $(12.1 \pm 2.1$ cells; total $n=291)$, to $9 \mathrm{~mm}(20.0 \pm 2.2$ cells total $n=$

\section{$\leftarrow$}

each ventral root recording). Respiration-modulated cells in the intact network could reliably be identified in relation to motor output, despite skipped cycles (shaded box, top left) and ectopic bursts (arrows, top right). CVs are displayed to the left of optical traces and vertical bars to the right scale relative luminance changes $(\Delta F / F)$; fine lines link traces obtained from the same neuron in different recording epochs. Heavy traces indicate neurons that remained synaptically coupled following synaptic blockade at $9 \mathrm{~mm}$ (left column) and $6 \mathrm{~mm}\left[\mathrm{~K}^{+}\right]_{0}$ (right column). These cell pairs are linked by black dotted lines with arrowheads in $\boldsymbol{A}$. 


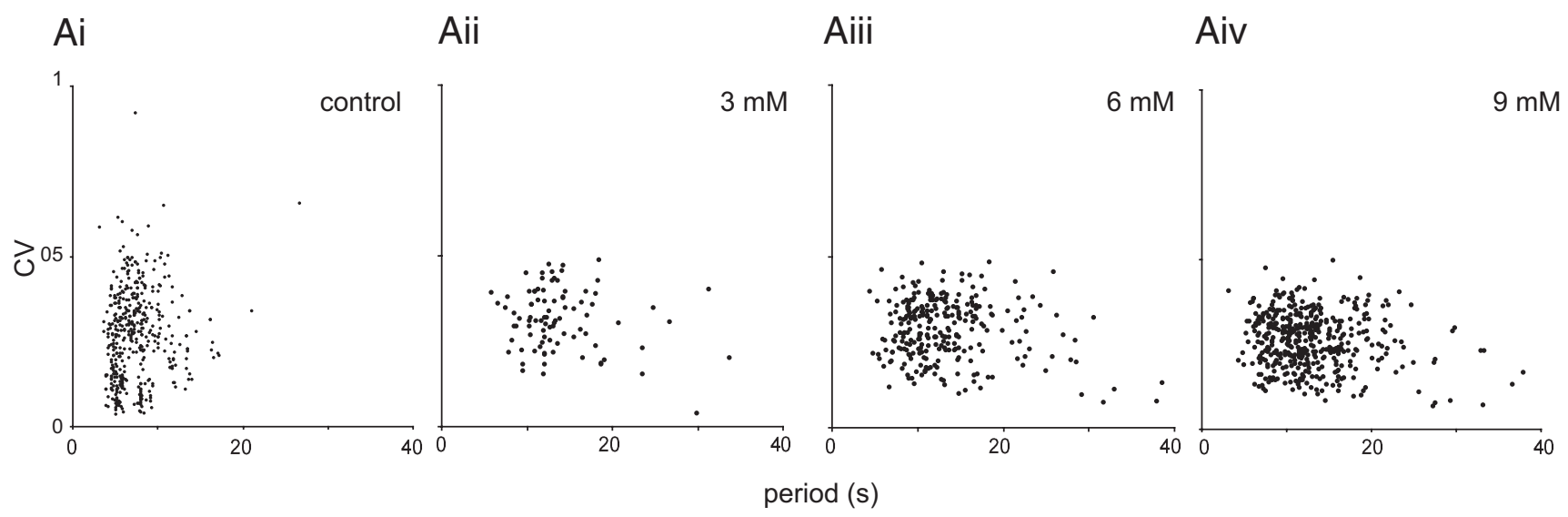

B

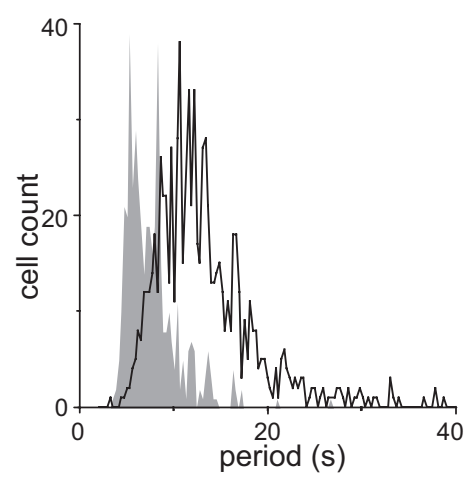

C

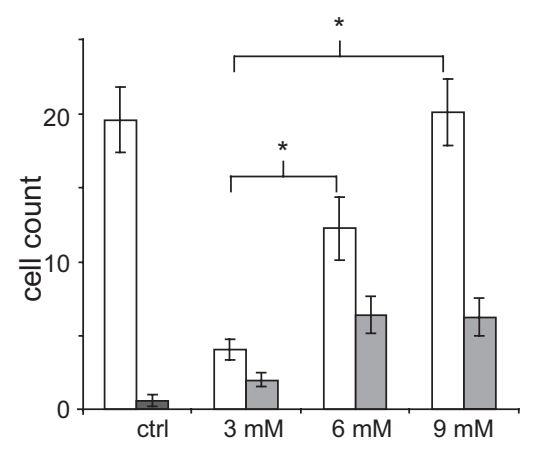

D

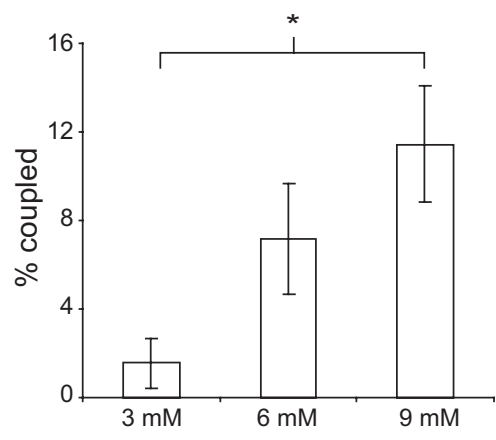

Figure 3. Statistics on pooled cycle periods. A, Scatter plots of periods $\times C V$ for respiration-modulated neurons in the intact network (Ai); and following blockade of fast synaptic transmission at $3 \mathrm{~mm}$ (Aii), $6 \mathrm{~mm}$ (Aiii), and $9 \mathrm{~mm}$ (Aiv) $\left[\mathrm{K}^{+}\right]_{0}$. Respiration-modulated neuron periods are more variable than EB periods because of skipped or ectopic bursts, and because only neurons with CV $<$ 0.5 were included as EBs. $\boldsymbol{B}$, Histogram of respiration-modulated neuron periods (solid gray) and EB periods show that EB periods were slower than respiration-modulated periods; this difference was statistically significant $(p<0.01)$.C, Average number of optically recorded cells in each recording epoch under control conditions and following synaptic blockade (hollow bars) reveals that EB numbers increased significantly with $\left[\mathrm{K}^{+}\right]_{0}$. At each $\left[\mathrm{K}^{+}\right]_{0}$, approximately half the units were active at another $\left[\mathrm{K}^{+}\right]_{0}$ (gray bars); only 2.8 respiration-modulated neurons per recording location were subsequently identified as EBs (black bar). $\boldsymbol{D}$, Using the average correlation coefficient between respiration-modulated neurons $\left(R^{2}=0.67\right)$ as the criterion, cells that remained coupled following synaptic blockade were identified; although their numbers increased with $\left[\mathrm{K}^{+}\right]_{0}$, only the difference between $3 \mathrm{~mm}$ and $9 \mathrm{~mm}\left[\mathrm{~K}^{+}\right]_{0}$ was statistically significant.

$450)$; these differences were statistically significant $(p<0.01$, with Bonferroni correction) (Fig. 3C). This increase is also apparent in EB totals at each $\left[\mathrm{K}^{+}\right]_{0}$, which increase monotonically from $3 \mathrm{~mm}(n=91)$ to $6 \mathrm{~mm}(n=291)$ to $9 \mathrm{~mm}(n=450)$; Although this increase is plausibly due to the transition from quiescence at low $\left[\mathrm{K}^{+}\right]_{\mathrm{o}}$ to bursting at higher $\left[\mathrm{K}^{+}\right]_{\mathrm{o}}$, it also likely overstates EB numbers, due to increased coupling detected at higher $\left[\mathrm{K}^{+}\right]_{\mathrm{o}}$. We found that the number of coupled bursters (as a percentage of all bursters) increased from $1.5 \pm 0.1 \%$ at $3 \mathrm{~mm}\left[\mathrm{~K}^{+}\right]_{\mathrm{o}}$ to $7.1 \pm 2.5 \%$ at $6 \mathrm{~mm}$, and $11.4 \pm 2.6 \%$ at $9 \mathrm{~mm}$ (Fig. $3 D$ ). Although only the difference between $3 \mathrm{~mm}$ and $9 \mathrm{mM}\left[\mathrm{K}^{+}\right]_{\mathrm{o}}$ was statistically significant $(p<$ 0.05, with Bonferroni correction) (Fig. $3 D$ ), the observed coupling between cells following synaptic blockade at higher $\left[\mathrm{K}^{+}\right]_{\mathrm{o}}$ suggests that at higher $\left[\mathrm{K}^{+}\right]_{\mathrm{o}}$, at least some of the rhythmically active cells follow, rather than spontaneously generate rhythmic activity. This observation suggests that at elevated $\left[\mathrm{K}^{+}\right]_{\mathrm{o}}$, with the synaptic isolation methods used here, the number of EBs is likely overestimated.

\section{Respiratory neurons and EBs form partially overlapping clusters}

A goal of this study was to characterize the anatomical distribution of EBs in relation to respiratory networks. To this end, datasets were aligned using the VIIc and the ventral surface as aligning landmarks (Fig. 4A, fine gray lines). Based on functionalanatomical studies (Smith et al., 1991; Ruangkittisakul et al.,
2008), the boundaries of the preBötC span $200 \mu \mathrm{m}$, centered 500 $\mu \mathrm{m}$ caudal to the VIIc; these anatomical boundaries are shown in Figure 4 as heavy broken lines.

Consistent with many earlier published reports, respiratory neurons (Fig. $4 \mathrm{~A}$, hollow black circles) were found all along the rostrocaudal extent of ventrolateral medulla, while EBs (Fig. $4 A$, hollow red circles) were relatively sparse caudal to the preBötC $(n=150)$. Respiration-modulated neurons that were later identified as EBs (filled black circles) were mainly found at or rostral to the preBötC. Although the overall proportion of respirationmodulated EBs to respiration-modulated neurons was $24 \%$ across all $\left[\mathrm{K}^{+}\right]_{\mathrm{o}}$, within the boundaries of the preBötC, this proportion was 55\% (28/51); in a $200 \mu \mathrm{m}$ window centered on VIIc, this proportion was $53 \%(23 / 43)$.

The $\chi^{2}$ test rejected the null hypothesis that the distribution of either respiratory neurons or EBs conformed to CSR $(p<0.001)$. To qualitatively describe clustering, density plots were generated using a $100 \mu \mathrm{m}$ Gaussian smoothing kernel. These plots revealed that points associated with respiratory neurons formed four clusters, within the VIIn and at the VIIc, two adjacent clusters within the preBötC, and a fourth cluster caudal and slightly dorsal to the preBötC (Fig. 4B). By contrast, EBs formed only three clusters, two partially merged clusters at the VIIc and extending dorsal and caudally, and a third cluster at the rostral margin of the preBötC (Fig. 4C). 
Because the shape and number of peaks in the density plot were sensitive to choice of smoothing kernel size, we used Dirichlet tessellations to identify statistically significant clusters. Statistically significant clusters (tiles and points outlined in black in Fig. $4 B$ and in red in Fig. $4 C$ ) superimpose onto peaks in the density plot. Applied to respiratory neurons, these methods identified three statistically significant clusters of $>8$ cells within, but at the caudal margin of the VIIn; two clusters of at least 16 contiguous cells at the rostral and caudal margins of the preBötC; finally, a third region containing two clusters of $>22$ cells extended caudally from $\sim 100 \mu \mathrm{m}$ caudal to the preBötC (Fig. 4B).

Statistically significant clusters of EBs were larger and fewer than those associated with respiratory neurons. The first was found at or just rostral to the VIIc, the second about $50 \mu \mathrm{m}$ caudal to the VIIc and slightly dorsal to the first cluster, each containing at least 21 cells; a third statistically significant cluster containing 43 cells was identified at the rostral margin of the preBötC (Fig. 4C). Although other Dirichlet tiles associated with both respiratory neurons and EBs that were significantly smaller than tiles generated by shuffled surrogate data were distributed along the neuraxis, because they were spatially isolated, they are not considered markers of statistically significant clusters.

To directly compare the distribution of respiratory neurons and EBs, outlines of the large statistically significant clusters in Figure 4, $B$ and $C$, are superimposed (Fig. $4 D$ ). Strikingly, respiratory neurons (Fig. $4 D$, blue outlines) and EBs (Fig. $4 D$, red outlines) only overlapped at the VIIc and at the rostral margin of the preBötC. These regions of overlap between statistically significant clusters of respiratory neurons and EBs, closely matched statistically significant clusters of respirationmodulated EBs (Fig. 4D, purple outlines; associated density plot not shown). In addition to these overlapping clusters, both EBs (between the VIIc and the preBötC) and respiratory neurons (caudal to the preBötC) form clusters with little or no overlap.

\section{Heterogeneous EB burst frequency response to $\left[\mathrm{K}^{+}\right]_{\mathrm{o}}$ elevation}

Subsets of EBs were active at more than one $\left[\mathrm{K}^{+}\right]_{\mathrm{o}}$ (Fig. $3 \mathrm{C}$ ). Because elevating $\left[\mathrm{K}^{+}\right]_{\mathrm{o}}$ depolarizes neurons (Del Negro et al., 2001), EBs whose bursting mechanism is voltage dependent would be expected to increase their bursting frequency with increasing $\left[\mathrm{K}^{+}\right]_{\mathrm{o}}$. As a consequence, EBs ac-
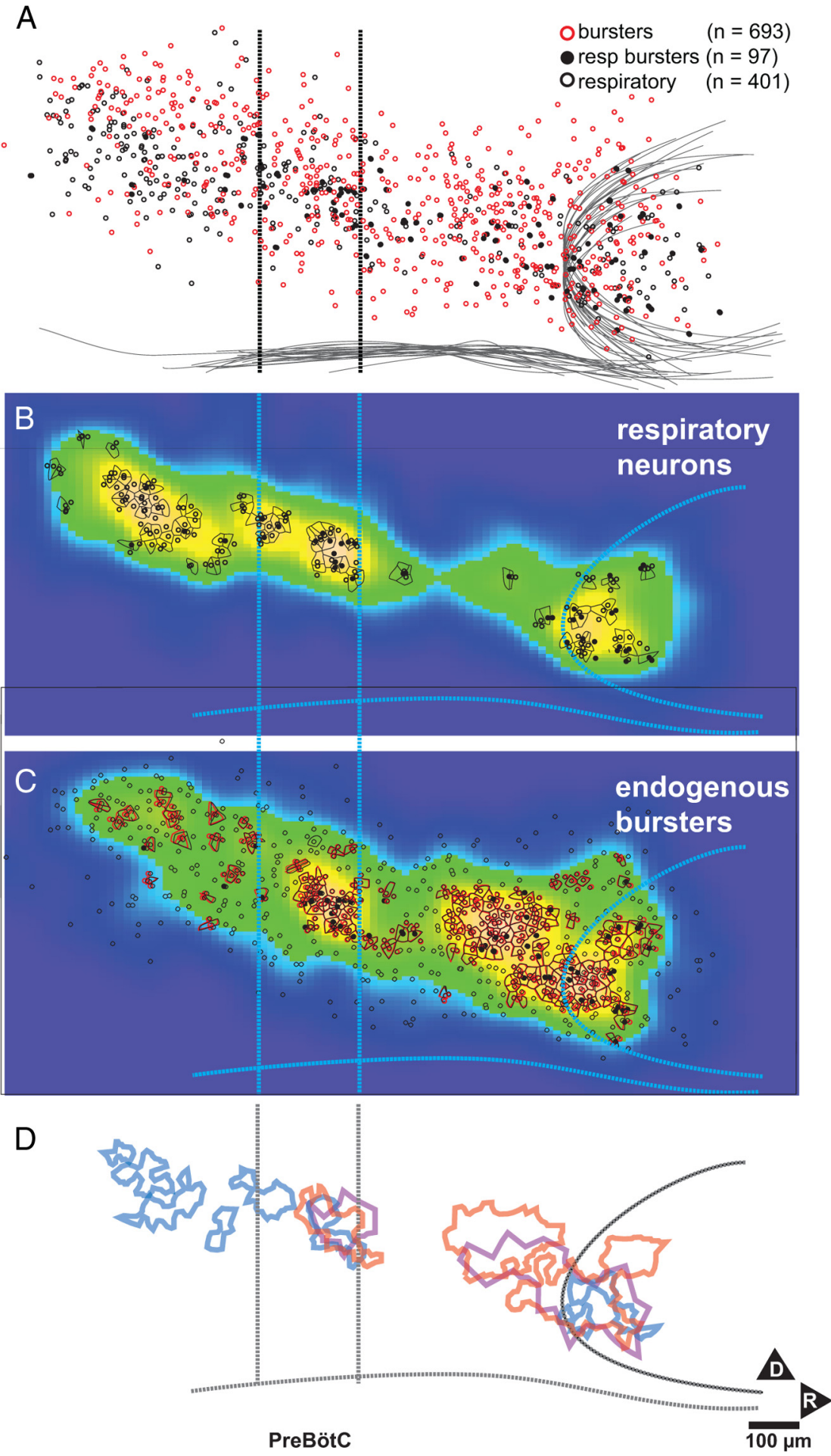

Figure 4. Respiratory neurons and EBs form overlapping clusters along ventrolateral medulla. $A$, Dot diagram shows distribution of all respiratory neurons (hollow black dots), EBs (hollow red dots), and respiratory neurons that were later identified as EBs (solid black dots). Fine lines show the outlines of VIIn and ventral surface; these landmarks were used to align datasets. Heavy vertical lines indicate the boundaries of the preBötC. $\boldsymbol{B}$, Respiratory neuron density plot generated with a $100 \mu \mathrm{m}$ smoothing kernel has peaks at the VIIC, within the preBötC, and $100 \mu \mathrm{m}$ caudal to the preBötC. Dirichlet tiles and dots are shown for those tiles significantly smaller than tiles generated from shuffled surrogate data. C, EB density plot has a large peak overlapping with the VIIC, and another at the rostral margin of the preBötc; tiles and associated dots significantly smaller than tiles from surrogate data are shown in red and solid black. $\boldsymbol{D}$, Statistically significant clusters, defined as regions containing at least eight contiguous Dirichlet tiles significantly smaller than tiles from shuffled surrogate data, for respiratory neurons (blue outlines), EBs (red outlines), and respiratory EBs (purple outlines). Statistically significant clusters of EBs and respiratory neurons overlap at the VIIc, and within the preBötC; in addition, a large cluster of EBs caudal to VIIn does not overlap with respiratory neurons, and a cluster of respiratory neurons caudal to the preBötC does not overlap with EBs. Regions of overlap between EB and respiratory neuron clusters closely match statistically significant clusters of respiratory EBs. 
$\mathrm{Ai}$

B

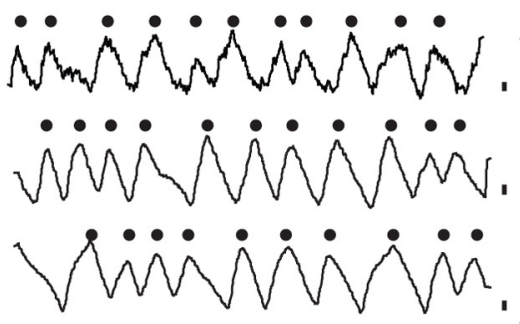

Aii

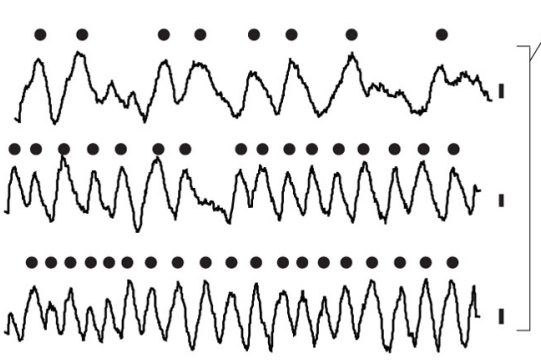

$\frac{110 \% \Delta \mathrm{F} / \mathrm{F}}{20 \mathrm{~s}}$

D

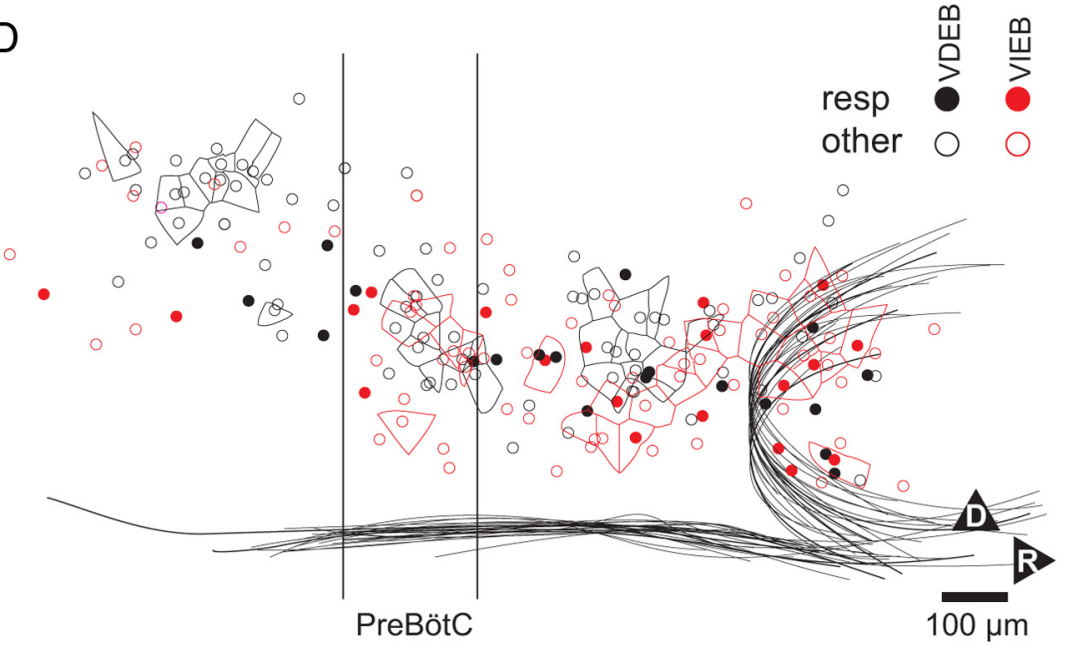

Figure 5. Changes in $\left[\mathrm{K}^{+}\right]_{0}$ had variable effects on EB burst frequency. $A$, Traces from two EBs active at 3, 6, and $9 \mathrm{~mm}\left[\mathrm{~K}^{+}\right]_{0}$ following blockade of fast synaptic transmission. The upper set of traces $(\boldsymbol{A} \boldsymbol{i})$ are from a neuron whose mean period does not decrease with increasing $\left[\mathrm{K}^{+}\right]_{0}$, referred to as VIEBs; the lower set of traces (Aii) are from a neuron whose mean period decreases with increasing $\left[\mathrm{K}^{+}\right]_{0}$, referred to as VDEBs. Dots indicate peaks used to calculate periods. $\boldsymbol{B}, \mathrm{ROl}$ recording locations at $3 \mathrm{~mm}$ (black circles), $6 \mathrm{~mm}$ (blue circles), and $9 \mathrm{~mm}$ (red circles) $\left[\mathrm{K}^{+}\right]_{0}$ in relation to the VIIn and brainstem ventral surface (black outlines). $C$, Although the relative numbers of VDEBs and VIEBs were not significantly different, the percentage of neurons active at more than one $\left[\mathrm{K}^{+}\right]_{0}$, and hence classified as either VDEB or VIEB, was significantly greater among respiration-modulated EBs than among EBs in general. $\boldsymbol{D}$, Dot diagram of VDEBs (black) and VIEBs (red), subdivided into respiratory (solid) or other (hollow). Statistically significant clusters of VDEBs and VIEBS (associated with at least 7 contiguous Dirichlet tiles) overlap within the preBötC; VIEBs are rare caudal to the preBötC, while VDEBs are relatively sparse at the level of the VIIn.

tive at more than one $\left[\mathrm{K}^{+}\right]_{\mathrm{o}}$ were classified as voltage-dependent EBs (VDEBs) or voltage-independent EBs (VIEBs), depending on whether burst frequency increased with $\left[\mathrm{K}^{+}\right]_{\mathrm{o}}$ or not. Traces from a VIEB (Fig. 5Ai) and a VDEB (Fig. 5Aii) both recorded slightly caudal to the VIIn (Fig. $5 B$ ) reveal that while in this VIEB, bursting frequency is unaffected by changes in $\left[\mathrm{K}^{+}\right]_{\mathrm{o}}$, bursting pattern is transformed from sharply rising and steeply decrementing at $3 \mathrm{~mm}\left[\mathrm{~K}^{+}\right]_{\mathrm{o}}$ to rounded in shape (Fig. $5 A i$, top trace vs bottom traces), consistent with near-tonic spiking. By contrast, in the VDEB shown here, while frequency increases with $\left[\mathrm{K}^{+}\right]_{\mathrm{o}}$, burst envelopes remain steeply rising and decrementing (Fig.
5Aii). The overall number of VIEBs and VDEBs were approximately equal, both as a fraction of total EBs and as a fraction of respiration-modulated EBs (Fig. 5C). The proportion of respiration-modulated EBs active at multiple $\left[\mathrm{K}^{+}\right]_{\mathrm{o}}(41 \% ; 40 / 97)$, was almost double that of all EBs $(26 \% ; 181 / 693)$.

By combining data across experiments in a dot diagram (Fig. 5D), a qualitative feature that emerges is that there are almost no VIEBs $(n=86)$ caudal to the preBötC, and few VDEBs $(n=95)$ rostral to VIIc. Using Dirichlet tiling to detect statistically significant clustering of VDEBs and VIEBs (tiles outlined in black and red, respectively; Fig. 5D), clusters associated with these two cell classes show little overlap: VIEBs cluster as a band along dorsal VIIn running caudally past VIIc, while VDEBs form one cluster just caudal to the VIEB cluster at the level of the VIIn, and another caudal to the preBötC. In addition to these non-overlapping clusters, smaller $(n>7)$ VIEB and VDEB clusters overlap at the rostral margin of the preBötC.

\section{Elevated $\left[\mathrm{K}^{+}\right]_{\mathrm{o}}$ induces ectopic respiratory neuron bursting and attenuated ventral root bursts in synaptically coupled respiratory networks}

Because the number of EBs increased significantly with $\left[\mathrm{K}^{+}\right]_{\mathrm{o}}$, and subsets of them have been found to be respiration modulated and voltage dependent, the presence of EBs within respiratory rhythmogenic networks is predicted to give rise to ectopic bursting among respiratory network constituents at elevated $\left[\mathrm{K}^{+}\right]_{\mathrm{o}}$. To test this conjecture, seven experiments were performed in which the synaptically coupled network was recorded at 3,6 , and $9 \mathrm{~mm}\left[\mathrm{~K}^{+}\right]_{0}$. Increases in $\left[\mathrm{K}^{+}\right]_{\mathrm{o}}$ modified both respiratory network activity and motor output. Respiratory network constituents increased between 3 and $9 \mathrm{mM}\left[\mathrm{K}^{+}\right]_{\mathrm{o}}$, from $20 \pm 9$ cells at $3 \mathrm{~mm}$, to $23 \pm 7$ cells at $6 \mathrm{~mm}$, and $35 \pm 9$ cells at $9 \mathrm{~mm}$ (Fig. $6 \mathrm{~A}$ ); repeated-measures ANOVA revealed statistically significant differences between 3 and $9 \mathrm{~mm}$ and between 6 and $9 \mathrm{~mm}(p<0.05)$. In addition, the period of respiratory motor output decreased with $\left[\mathrm{K}^{+}\right]_{\mathrm{o}}$ (Fig. $6 \mathrm{~B}$, top, hollow black squares). Period variability within each recording epoch, estimated using the mean of the CVs also increased with $\left[\mathrm{K}^{+}\right]_{\mathrm{o}}$ (Fig. 6, bottom, hollow black squares).

Across experiments, a total of 44 cells were active at all three $\left[\mathrm{K}^{+}\right]_{\mathrm{o}}$ levels; the activity of these cells was analyzed in greater detail to quantify change in ectopic activity. Using repeatedmeasures ANOVA, mean cell periods at $6 \mathrm{~mm}$ were found to be significantly shorter than at 3 and $9 \mathrm{~mm}(p<0.01)$ (Fig. $6 B$, top, hollow red circles). Period variability (estimated using the mean of CVs under each $\left[\mathrm{K}^{+}\right]_{\mathrm{o}}$ ) increased with $\left[\mathrm{K}^{+}\right]_{\mathrm{o}}$, and the $\mathrm{CV}$ at 9 $\mathrm{mm}$ was significantly greater than at $3 \mathrm{~mm}(p=0.02)$ (Fig. 6 , 
bottom, hollow red circles). Accurate estimate of respiratory neuron period was complicated by increasing peak height variability with $\left[\mathrm{K}^{+}\right]_{\mathrm{o}}$, apparent both in the motor output (attenuated bursts indicated by hollow arrows; Fig. 6D) and in the associated optical traces (Fig. 6D) obtained from one set of recordings from VIIn (Fig. 6C). As can be seen from the optical traces, although ectopic bursts (Fig. 6D, solid red arrowheads) occur at 3 $\mathrm{mm}\left[\mathrm{K}^{+}\right]_{\mathrm{o}}$, their frequency increases with increasing $\left[\mathrm{K}^{+}\right]_{\mathrm{o}}$. Burst triggered averages (Fig. $6 E$ ) show that the phase of respiratory neuron activity at $3 \mathrm{~mm}\left[\mathrm{~K}^{+}\right]_{\mathrm{o}}$ (black lines) can be substantially altered at $6 \mathrm{~mm}$ (Fig. $6 E$, blue lines) and at $9 \mathrm{~mm}$ (Fig. $6 \mathrm{E}$, red lines); phase of activity can become so variable as to flatten the burst-triggered average (cells 1 and 3 ), suggesting that in this preparation, 9 $\mathrm{mm}\left[\mathrm{K}^{+}\right]_{\mathrm{o}}$ disrupts respiratory rhythmogenic network function. Ectopic bursting at the cellular level is the expected effect of elevated $\left[\mathrm{K}^{+}\right]_{\mathrm{o}}$ on VDEBs, and the increased variability of motor output amplitude and frequency is consistent with these neurons playing a rhythmogenic role (Onimaru and Homma, 2008).

\section{Discussion}

In this study, optical recordings characterized the overlap between respiration-modulated neurons and EBs in ventrolateral medulla. The sample size provides robust support for two main findings: both respiratory networks and EBs form clusters that overlap in regions identified as rhythmogenic; and $\mathrm{EB}$ numbers show a steep dependence on $\left[\mathrm{K}^{+}\right]_{\mathrm{o}}$, with relatively few active at physiological $(3 \mathrm{~mm})\left[\mathrm{K}^{+}\right]_{\mathrm{o}}$.

\section{Anatomical overlap between respiratory networks and EBs suggests functional specialization}

The anatomical distribution of respiratory neurons is consistent with earlier studies. Respiratory neurons were found along the caudal, ventral, and dorsal margins of VIIn. These neurons formed a significant cluster at the VIIc, which overlaps with the putatively rhythmogenic RTN/pFRG. This structure includes neurons developmentally specified by the transcription factor phox $2 b$ (Onimaru et al., 2009; Thoby-Brisson et al., 2009), that are distributed along the ventrolateral margin of the VIIn, curving medially at the VIIc, and that have also been shown to play a key role in central chemosensation (Lazarenko et al., 2009; Guyenet and Mulkey, 2010). Likewise, the preBötC has been identified as a $200 \mu \mathrm{m}$ region centered $500 \mu \mathrm{m}$ caudal to the VIIc (Ruangkittisakul et al., 2008), which in our data coincides with a statistically significant cluster of respirationmodulated neurons. Finally, a significant cluster of respirationmodulated neurons lying caudal and dorsal to the preBötC was found, consistent with relay neurons in the ventral respiratory group (Dobbins and Feldman, 1994). Although in any given experiment only neurons at the surface were recorded from, across experiments the respiratory column was transected at different mediolateral lev-
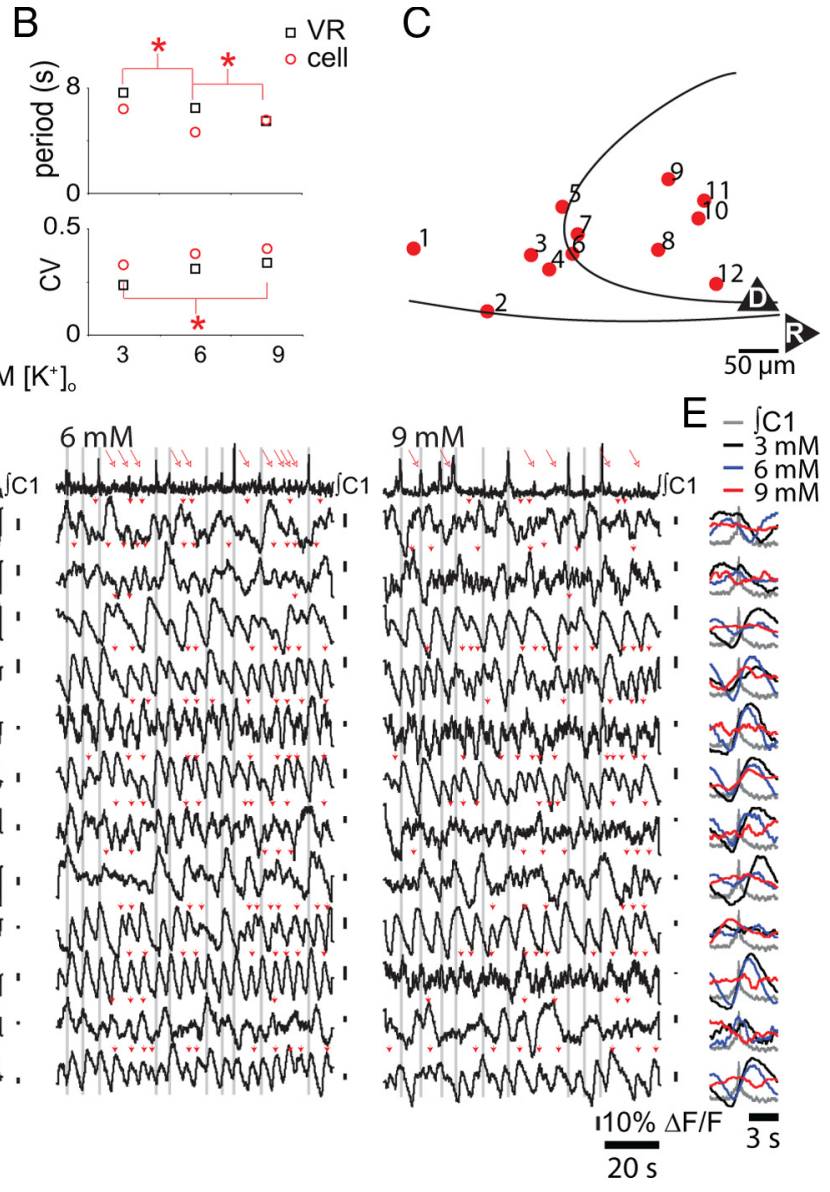

Figure 6. Changes in $\left[\mathrm{K}^{+}\right]_{0}$ in synaptically coupled respiratory networks increases the number of respiratory network constituents, and increases respiratory variability, at both the cellular and systems level. $\boldsymbol{A}$, Mean number of respiration-modulated increases with $\left[\mathrm{K}^{+}\right]_{0}$. B , Top, Mean ventral root (hollow black squares; VR) and cell (hollow red circles) periods. Cell 知 $]_{0}$ were significantly shorter than cell periods at 3 or $9 \mathrm{~mm}\left[\mathrm{~K}^{+}\right]_{0}$ (red asterisks). Bottom, Mean VR and cell CV $\left.{ }^{+}\right]_{0}$ increased, motor output amplitude and frequency became less regular; truncated inspiratory bursts are indicated by red

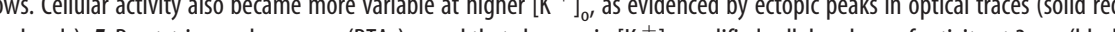
arrowheads). $\boldsymbol{E}$, Burst-triggered averages (BTAs) reveal that changes in $\left[\mathrm{K}^{+}\right]_{0}$ modified cellular phase of activity at $3 \mathrm{~mm}$ (black lines), $6 \mathrm{~mm}$ (blue lines), and $9 \mathrm{~mm}$ (red lines) $\left[\mathrm{K}^{+}\right]_{0}$, relative to the ventral root burst (gray line).

els, due to experimenter error and/or biological variability. Thus these data aggregate respiratory network activity from a range of mediolateral levels. Further, because putatively rhythmogenic glutamatergic neurons coexpressing the neurokinin-1 receptor and somatostatin within the preBötC are confined to a narrow band of cells in the mediolateral axis (Stornetta et al., 2003), available data suggest that, at least at the level of the preBötC, rhythmogenic networks show little mediolateral dispersion.

Less is known about the distribution of EBs in ventrolateral medulla. Based on single-unit (Onimaru et al., 1989; Johnson et al., 1994; Onimaru et al., 1995; Del Negro et al., 2002a) and optical (Koshiya and Smith, 1999; Koizumi et al., 2008) recordings, both the RTN/pFRG and the preBötC have been shown to be rich in EBs. These studies left unresolved the distribution of EBs along ventrolateral medulla. If EBs were found to be uniformly distributed, or if the overlap between EBs and respiratory networks was small, this would undermine the hypothesis that EBs represented functionally specialized respiratory network constituents. The novel and-because of the relatively large sample size-robust finding of this study is that, while both respiration-modulated 
neurons and EBs are distributed along the length of ventrolateral medulla, statistically significant clusters of these two classes of neurons only overlap at the VIIc and within the preBötC, both identified loci of respiratory rhythmogenesis. This overlap is also apparent in the high proportion of respiration-modulated EBs to respirationmodulated neurons within the preBötC and in VIIc, consistent with an earlier study (Koizumi et al., 2008). Another feature of respirationmodulated EBs was that the proportion of EBs active at more than one $\left[\mathrm{K}^{+}\right]_{\mathrm{o}}$ was near twice that for EBs as a group. These features are consistent with a functional role for EBs within rhythmogenic networks.

\section{On the role of EBs in respiratory rhythm generation and regulation}

In the transverse slice, respiratory rhythm persists after pharmacological disruption of the biophysical mechanism for bursting prevalent in preBötC neurons in perinatal rodents (Del Negro et al., 2002a; discrepant with Koizumi and Smith, 2008). Based on these findings, the obligatory role of EBs in respiratory rhythmogenesis has been challenged (Feldman and Del Negro, 2006; Del Negro et al., 2008). Evidence that two distinct functional anatomical regions are rhythmogenic (Mellen et al., 2003; Dubreuil et al., 2009; Onimaru et al., 2009) establishes that respiratory rhythm can arise via more than one mechanism. Thus, generically, the observation that a given structure or mechanism is not obligatory for a behavior does not support the inference that the structure or mechanism plays no role in that behavior. A similar point has been made about the role of EBs in respiratory rhythmogenesis (Ramirez et al., 2004). The appearance of ectopic peaks in respiratory neuron traces, together with increased variability in inspiratory burst amplitude and respiratory period at elevated $\left[\mathrm{K}^{+}\right]_{\mathrm{o}}$ under network-intact conditions, is consistent with EBs contributing to respiratory rhythmogenesis.

Although the anatomical distribution of EBs is consistent with rhythmogenic function, the observed steep dependence of EB numbers on $\left[\mathrm{K}^{+}\right]_{\mathrm{o}}$ suggests that under physiological conditions, the contribution of EBs is dependent on the level of excitatory drive. The relatively low number of EBs active at physiological $\left[\mathrm{K}^{+}\right]_{\mathrm{o}}$ does not rule out rhythmogenic function, however, since network models have shown that EB-driven network bursting can be obtained in a network in which as few as $10 \%$ of its constituents are rhythmically active (Butera et al., 1999b). In addition to a possible direct role, the presence of quiescent conditional bursters in respiratory networks may contribute to rhythmogenesis at the network level, via nonlinear amplification of synaptic drive via the same biophysical mechanisms that produce bursting at more depolarized membrane potentials (Feldman and Del Negro, 2006), as has been shown in modeling studies (Butera et al., 1999b; Purvis et al., 2007). A corollary to this is that mechanisms for rhythmogenesis may be qualitatively different at physiological $\left[\mathrm{K}^{+}\right]_{\mathrm{o}}(3 \mathrm{~mm})$ than at the elevated $\left[\mathrm{K}^{+}\right]_{\mathrm{o}}(9 \mathrm{mM})$ routinely used in vitro, since at elevated $\left[\mathrm{K}^{+}\right]_{\mathrm{o}}$, more conditional bursters will enter into a bursting regime, potentially transforming the contribution of EBs from primarily amplifiers of synaptic drive to sources of rhythmic activation of the networks in which they are embedded.

Despite the overlap between EB and respiratory neuron clusters, most EBs were not respiration modulated under control conditions. Because descending and afferent inputs have been removed in vitro, and cells at the sliced surface have lost processes and synaptic inputs, active in vitro networks likely represent a fraction of those active in vivo. The significant increase in respiration-modulated neurons as $\left[\mathrm{K}^{+}\right]$o was elevated from $3 \mathrm{~mm}$ to $9 \mathrm{~mm}$ in synaptically coupled networks supports this conjecture. As a consequence, the observation that most EBs lacked respiratory modulation provides only weak support for the inference that they are nonrespiratory. More accurate quantification of nonrespiratory EBs awaits characterization of respiratory network phenotypes, so that more robust exclusion criteria can be applied.

\section{Methodological considerations}

In the experiments performed here, $\left[\mathrm{K}^{+}\right]_{\mathrm{o}}$ was manipulated to shift membrane potential, and hence manipulate neuronal excitability. In a single-unit recording study taking a similar approach, EB bursting frequency was found to be steeply dependent on $\left[\mathrm{K}^{+}\right]_{\mathrm{o}}$, and no VIEBs were described (Del Negro et al., 2001). In this study however, a robust effect of $\left[\mathrm{K}^{+}\right]_{\mathrm{o}}$ on frequency was obtained under conditions of excitatory transmission blockade only. In another study in which both fast excitatory and inhibitory transmission were blocked, $\mathrm{EB} V_{\mathrm{m}}$ was found to be insensitive to $\left[\mathrm{K}^{+}\right]_{\mathrm{o}}$ manipulation because of the presence of a background $\mathrm{Na}^{+}$ conductance (Tryba et al., 2003); thus, neurons matching the VDEBs in our study were not encountered. These studies, as well as our own data, suggest that EBs have heterogeneous biophysical properties, a feature that has been shown to extend the dynamic range of rhythmogenic networks (Butera et al., 1999b).

Optical recordings revealed that subsets of EBs remained coupled following synaptic blockade. Such coupling would be expected to persist between gap-junction-coupled neurons, and gap junction coupling between respiratory network constituents both has been observed (Rekling et al., 2000; Thoby-Brisson et al., 2009) and would be expected in tissue harvested from neonates (Kandler and Katz, 1995). More likely though, given the large number of metabotropic and/or ionotropic conductances left unblocked using our cocktail, coupling is at least in part mediated by chemical synapses. As with other studies in which synaptic blockade is implemented via by blocking fast excitatory (Koshiya and Smith, 1999; Del Negro et al., 2001, 2002b; Thoby-Brisson and Ramirez, 2001; Koizumi and Smith, 2008; Koizumi et al., 2008) or fast excitatory and inhibitory (Tryba et al., 2003; Peña et al., 2004; Del Negro et al., 2005; Zavala-Tecuapetla et al., 2008) transmission, synaptic transmission is more accurately described as attenuated than blocked. Other approaches such as low $\left[\mathrm{Ca}^{2+}\right]_{\mathrm{o}}$ (Onimaru et al., 1989; Johnson et al., 1994; Onimaru et al., 1995; Del Negro et al., 2001, 2002a), $\mathrm{Cd}^{2+}$ blockade of $\mathrm{Ca}^{2+}$ channels (Del Negro et al., 2002b; Koizumi and Smith, 2008; Koizumi et al., 2008), or $\mathrm{Na}^{+}$channel blockade via tetrodotoxin are each problematic because they may disrupt the biophysical mechanisms that give rise to bursting. Another approach, depletion of vesicular stores via vacuolar proton ATPase blockade (Cavelier and Attwell, 2007), effectively blocks synaptic release with less impact on biophysics, but is too slow to be practicable in most cases. Insofar as nonbursters are included in our EB sample, however, the robust chemical coupling that yokes them to EBs may render them functionally equivalent to the EBs that drive them.

\section{Conclusion}

Here, evidence has been presented suggesting that EBs are constituents of respiratory rhythmogenic networks at the level of the RTN/pFRG and preBötC. Characterizing the role or roles of EBs in respiratory rhythmogenesis is challenging, because anatomically distributed heterogenous networks in ventrolateral medulla interact to generate respiratory rhythm and regulate it from breath to breath. Because optical recording methods offer access to portions of these networks in parallel, they provide a powerful tool for elucidating cellular and synaptic mechanisms underpinning this rich and complex behavior. 


\section{References}

Baddeley A, Turner R (2005) Spatstat: an R package for analyzing spatial point patterns. J Stat Softw 12:1-42.

Barnes BJ, Tuong CM, Mellen NM (2007) Functional imaging reveals respiratory network activity during hypoxic and opioid challenge in the neonate rat tilted sagittal slab preparation. J Neurophysiol 97:2283-2292.

Birchmeier C, Brunet JF, Fortin G, Goridis C (2009) Defective respiratory rhythmogenesis and loss of central chemosensitivity in Phox $2 \mathrm{~b}$ mutants targeting retrotrapezoid neurons. J Neurosci 29:14836-14846.

Butera RJ Jr, Rinzel J, Smith JC (1999a) Models of respiratory rhythm generation in the pre-Botzinger complex. I. Bursting pacemaker neurons. J Neurophysiol 82:382-397.

Butera RJ Jr, Rinzel J, Smith JC (1999b) Models of respiratory rhythm generation in the pre-Botzinger complex. II. Populations of coupled pacemaker neurons. J Neurophysiol 82:398-415.

Cavelier P, Attwell D (2007) Neurotransmitter depletion by bafilomycin is promoted by vesicle turnover. Neurosci Lett 412:95-100.

Del Negro CA, Johnson SM, Butera RJ, Smith JC (2001) Models of respiratory rhythm generation in the pre-Botzinger complex. III. Experimental tests of model predictions. J Neurophysiol 86:59-74.

Del Negro CA, Morgado-Valle C, Feldman JL (2002a) Respiratory rhythm: an emergent network property? Neuron 34:821-830.

Del Negro CA, Koshiya N, Butera RJ Jr, Smith JC (2002b) Persistent sodium current, membrane properties and bursting behavior of pre-Botzinger complex inspiratory neurons in vitro. J Neurophysiol 88:2242-2250.

Del Negro CA, Morgado-Valle C, Hayes JA, Mackay DD, Pace RW, Crowder EA, Feldman JL (2005) Sodium and calcium current-mediated pacemaker neurons and respiratory rhythm generation. J Neurosci 25:446-453.

Del Negro CA, Pace RW, Hayes JA (2008) What role do pacemakers play in the generation of respiratory rhythm? Adv Exp Med Biol 605:88-93.

Dobbins EG, Feldman JL (1994) Brainstem network controlling descending drive to phrenic motoneurons in rat. J Comp Neurol 347:64-86.

Duyckaerts C, Godefroy G (2000) Voronoi tessellation to study the numerical density and the spatial distribution of neurones. J Chem Neuroanat 20:83-92.

Eugenin J, Nicholls JG, Cohen LB, Muller KJ (2006) Optical recording from respiratory pattern generator of fetal mouse brainstem reveals a distributed network. Neuroscience 137:1221-1227.

Feldman JL, Del Negro CA (2006) Looking for inspiration: new perspectives on respiratory rhythm. Nat Rev Neurosci 7:232-242.

Funke F, Dutschmann M, Muller M (2007) Imaging of respiratory-related population activity with single-cell resolution. Am J Physiol Cell Physiol 292: C508-C516.

Guyenet PG, Mulkey DK (2010) Retrotrapezoid nucleus and parafacial respiratory group. Respir Physiol Neurobiol. Advance online publication. Retrieved April 23, 2010. doi:10.1016/j.resp.2010.02.005.

Johnson SM, Smith JC, Funk GD, Feldman JL (1994) Pacemaker behavior of respiratory neurons in medullary slices from neonatal rat. J Neurophysiol 72:2598-2608.

Kandler K, Katz LC (1995) Neuronal coupling and uncoupling in the developing nervous system. Curr Opin Neurobiol 5:98-105.

Koizumi H, Smith JC (2008) Persistent $\mathrm{Na}^{+}$and $\mathrm{K}^{+}$-dominated leak currents contribute to respiratory rhythm generation in the pre-Botzinger complex in vitro. J Neurosci 28:1773-1785.

Koizumi H, Wilson CG, Wong S, Yamanishi T, Koshiya N, Smith JC (2008) Functional imaging, spatial reconstruction, and biophysical analysis of a respiratory motor circuit isolated in vitro. J Neurosci 28:2353-2365.

Koshiya N, Smith JC (1999) Neuronal pacemaker for breathing visualized in vitro. Nature 400:360-363.

Kosmidis EK, Pierrefiche O, Vibert JF (2004) Respiratory-like rhythmic activity can be produced by an excitatory network of non-pacemaker neuron models. J Neurophysiol 92:686-699.

Lazarenko RM, Milner TA, Depuy SD, Stornetta RL, West GH, Kievits JA, Bayliss DA, Guyenet PG (2009) Acid sensitivity and ultrastructure of the retrotrapezoid nucleus in Phox2b-EGFP transgenic mice. J Comp Neurol 517:69-86.

Mellen NM (2008) A vibrating microtome attachment for cutting brain slice preparations at reproducible compound angles relative to the midline. J Neurosci Methods 168:113-118.

Mellen NM, Tuong CM (2009) Semi-automated region of interest generation for the analysis of optically recorded neuronal activity. Neuroimage 47:1331-1340.

Mellen NM, Janczewski WA, Bocchiaro CM, Feldman JL (2003) Opioid- induced quantal slowing reveals dual networks for respiratory rhythm generation. Neuron 37:821-826.

Okabe A, Boots B, Sugihara K (1992) Spatial tessellations. Concepts and applications of Voronoi diagrams. Chichester, UK: Wiley.

Onimaru H, Homma I (2008) Two modes of respiratory rhythm generation in the newborn rat brainstem-spinal cord preparation. Adv Exp Med Biol 605:104-108.

Onimaru H, Arata A, Homma I (1989) Firing properties of respiratory rhythm generating neurons in the absence of synaptic transmission in rat medulla in vitro. Exp Brain Res 76:530-536.

Onimaru H, Arata A, Homma I (1995) Intrinsic burst generation of preinspiratory neurons in the medulla of brainstem-spinal cord preparations isolated from newborn rats. Exp Brain Res 106:57-68.

Onimaru H, Ikeda K, Kawakami K (2009) Phox2b, RTN/pFRG neurons and respiratory rhythmogenesis. Respir Physiol Neurobiol 168:13-18.

Pace RW, Del Negro CA (2008) AMPA and metabotropic glutamate receptors cooperatively generate inspiratory-like depolarization in mouse respiratory neurons in vitro. Eur J Neurosci 28:2434-2442.

Pace RW, Mackay DD, Feldman JL, Del Negro CA (2007) Role of persistent sodium current in mouse preBotzinger complex neurons and respiratory rhythm generation. J Physiol 580:485-496.

Peña F, Parkis MA, Tryba AK, Ramirez JM (2004) Differential contribution of pacemaker properties to the generation of respiratory rhythms during normoxia and hypoxia. Neuron 43:105-117.

Purvis LK, Smith JC, Koizumi H, Butera RJ (2007) Intrinsic bursters increase the robustness of rhythm generation in an excitatory network. J Neurophysiol 97:1515-1526.

Ramirez JM, Tryba AK, Peña F (2004) Pacemaker neurons and neuronal networks: an integrative view. Curr Opin Neurobiol 14:665-674.

Rekling JC, Feldman JL (1998) PreBotzinger complex and pacemaker neurons: hypothesized site and kernel for respiratory rhythm generation. Annual Rev Physiol 60:385-405.

Rekling JC, Shao XM, Feldman JL (2000) Electrical coupling and excitatory synaptic transmission between rhythmogenic respiratory neurons in the preBotzinger complex. J Neurosci 20:RC113(1-5).

Ruangkittisakul A, Schwarzacher SW, Secchia L, Poon BY, Ma Y, Funk GD, Ballanyi K (2006) High sensitivity to neuromodulator-activated signaling pathways at physiological $\left[\mathrm{K}^{+}\right]$of confocally imaged respiratory center neurons in on-line-calibrated newborn rat brainstem slices. J Neurosci 26:11870-11880.

Ruangkittisakul A, Schwarzacher SW, Secchia L, Ma Y, Bobocea N, Poon BY, Funk GD, Ballanyi K (2008) Generation of eupnea and sighs by a spatiochemically organized inspiratory network. J Neurosci 28:2447-2458.

Rubin JE, Hayes JA, Mendenhall JL, Del Negro CA (2009) Calcium-activated nonspecific cation current and synaptic depression promote networkdependent burst oscillations. Proc Natl Acad Sci U S A 106:2939-2944.

Smith JC, Feldman JL (1987) In vitro brainstem-spinal cord preparations for study of motor systems for mammalian respiration and locomotion. J Neurosci Methods 21:321-333.

Smith JC, Ellenberger HH, Ballanyi K, Richter DW, Feldman JL (1991) PreBötzinger complex: a brainstem region that may generate respiratory rhythm in mammals. Science 254:726-729.

Stornetta RL, Rosin DL, Wang H, Sevigny CP, Weston MC, Guyenet PG (2003) A group of glutamatergic interneurons expressing high levels of both neurokinin-1 receptors and somatostatin identifies the region of the pre-Botzinger complex. J Comp Neurol 455:499-512.

Suzue T (1984) Respiratory rhythm generation in the in vitro brain stemspinal cord preparation of the neonatal rat. J Physiol 354:173-183.

Thoby-Brisson M, Ramirez JM (2001) Identification of two types of inspiratory pacemaker neurons in the isolated respiratory neural network of mice. J Neurophysiol 86:104-112.

Thoby-Brisson M, Karlén M, Wu N, Charnay P, Champagnat J, Fortin G (2009) Genetic identification of an embryonic parafacial oscillator coupling to the preBotzinger complex. Nat Neurosci 12:1028-1035.

Thomas D, Tovey SC, Collins TJ, Bootman MD, Berridge MJ, Lipp P (2000) A comparison of fluorescent $\mathrm{Ca} 2+$ indicator properties and their use in measuring elementary and global Ca2 + signals. Cell Calcium 28:213-223.

Tryba AK, Peña F, Ramirez JM (2003) Stabilization of bursting in respiratory pacemaker neurons. J Neurosci 23:3538-3546.

Zavala-Tecuapetla C, Aguileta MA, Lopez-Guerrero JJ, González-Marín MC, Peña F (2008) Calcium-activated potassium currents differentially modulate respiratory rhythm generation. Eur J Neurosci 27:2871-2884. 